\title{
Original Paper \\ Acnistus arborescens (Solanaceae): an important food resource for birds in an Atlantic Forest site, Southeastern Brazil
}

\author{
Izar Araujo Aximoff ${ }^{1,4,5}$, Hudson Martins Soares ${ }^{2}$ \& Gabriel Bernadello ${ }^{3}$
}

\begin{abstract}
Biotic interactions related to the consumption of floral nectar and fruits as food resource by birds promoting plant pollination and dispersal, are essential for forest ecosystem functioning. The daily interaction of birds with Acnistus arborescens (Solanaceae), a shrub-tree, was studied in the Itatiaia National Park and its surroundings. Reproductive phenology was monthly monitored in eighteen individuals in 2015. During flowering (August to November) and fruiting (September to December) the interaction birds-plant was studied. Flowers were visited by seven hummingbird species and several insects. Fruit set was $87.3 \%$. Thirty-five bird species of eleven families consumed its fruit. Thraupidae with sixteen bird species was the family responsible for most of its frugivory $(53.9 \%)$. Considering that more than $10 \%$ of the birds richness of Itatiaia National Park INP interacts with $A$. arborescens, it can be considered an important plant species in maintaining local bird diverstity. Thus, for attracting many birds, A. arborescens can be used in the process of recovery of degraded areas, and also as a focal plant species for environmental education programs and birdwatching.
\end{abstract}

Key words: birds, frugivory, Itatiaia National Park, nectarivory.

\section{Resumo}

Interações bióticas relacionadas ao consumo de néctar e frutos como recurso alimentar de aves promovendo polinização e dispersão de plantas, são essenciais para o funcionamento do ecossistema florestal. A interação diária de pássaros com Acnistus arborescens (Solanaceae) foi estudada no interior e entorno do Parque Nacional do Itatiaia. A fenologia reprodutiva foi monitorada mensalmente em dezoito indivíduos durante o ano de 2015. Durante a floração (agosto a novembro) e frutificação (setembro a dezembro) foram estudadas as interações aves-planta. Flores foram visitadas por sete espécies de beija-flores e vários insetos. Quase 90\% das flores estudadas tornaram-se frutos. Trinta e cinco espécies de aves, pertencentes a onze famílias, foram consumidores dos frutos. Thraupidae com dezesseis espécies de aves foi a família responsável pela maior parte desse consumo (53,9\%). Considerando que mais de $10 \%$ da riqueza das aves do Parque Nacional do Itatiaia interagem com $A$. arborescens, essa espécie pode ser considerada como de grande importancia na manutenção da diversidade de aves. Essa característica de atração de aves, faz com que $A$. arborescens seja uma espécie a ter seu uso considerado em projetos de recuperação áreas degradadas, e também como espécie vegetal focal para programas de educação ambiental voltados para observação de aves.

Palavras-chave: aves, frugivoria, Parque Nacional do Itatiaia, nectarivoria.

\section{Introduction}

Biotic interactions such pollination and seed dispersal are vital ecological processes for forest ecosystem functioning and recover from disturbances (Reis et al. 1999; Roberts \& Gandolfi
2000; Silva et al. 2002; Ceccon \& Varassin 2014). Frugivory, for instance, is an animal-plant interaction considered critical to seed dispersal and recruitment of several plant species (Jordano et al. 2006; Côrtes et al. 2009; Novoa et al. 2011;

\footnotetext{
Instituto de Pesquisas Jardim Botânico do Rio de Janeiro - JBRJ, R. Pacheco Leão 915, Horto, 22460-030, Rio de Janeiro, RJ, Brazil.

${ }^{2}$ Associação Educacional Dom Bosco - AEDB, Curso de Ciências Biológicas, Av. Prof. Antonio Esteves 1, Campo de Aviação, 27523-000, Resende, RJ, Brazil.

${ }^{3}$ Universidad Nacional de Córdoba - CONICET, Inst. Multidisciplinario de Biología Vegetal - IMBIV, C.C. 495, 5000, Córdoba, Argentina.

${ }^{4}$ ORCID: <https://orcid.org/0000-0002-5502-2421>

${ }^{5}$ Author for correspondence: izar.aximoff@gmail.com
} 
Pizo 2012; Parrini \& Pacheco 2014). The diversity of interacting animal species and their foraging efficiency are directly influenced by both quantity and quality of resources offered by different plant species (Malizia 2001; Moegenburg \& Levey 2003; Aximoff \& Freitas 2009). Thus, plant species that provide resources for sustaining a wide animal species assemblage are extremely important for the conservation of ecosystems (Silva et al. 2002; Ceccon \& Varassin 2014).

In Neotropical forests, many Solanaceae members stand out by having an association with several frugivorous bird and mammal species (Albuquerque et al. 2006). This is one of the most representative families in the Brazilian Atlantic Forest (Oliveira-Filho \& Fontes 2000). For instance, Acnistus arborescens (L.) Schltdl. was considered a key species to the functioning of the forest ecosystem providing fruits that are eaten by bats (Engriser 1995; Novoa et al. 2011), marsupials (Cáceres \& Lessa 2012) and birds (Cross 1981; Wheelwright et al. 1984; Athiê \& Dias 2012; Verçoza et al. 2012). Besides that, the nectar of its flowers is also another resource consumed by animals of different groups of insects (Cocucci 2000; Smith et al. 2008) and also hummingbirds (Verçoza et al. 2012).

Considering the prevalence of bird consumption of Solanaceae fruits (Albuquerque et al. 2006), one of the most appropriate locations to study this interaction is the Itatiaia National Park (INP), pointed out as one of the areas with the highest diversity of avifauna in the Atlantic Forest (Bencke et al. 2006; ICMBio 2013). Pineschi (1990) recorded more than a hundred bird species as dispersers of seven Rapanea species (Myrsinaceae) in the INP. Additionally several studies on animalplant interactions have been conducted in this area, primarily related to reproductive biology of plant species pollinated by hummingbirds (e.g., Canela 2006; Aximoff \& Freitas 2010; Wolowski et al. 2013a, b), birds (Aximoff \& Freitas 2009) and insects (Pires \& Freitas 2008; Wolowski \& Freitas 2010; Avila Jr. \& Freitas 2011; Fonseca 2012; Freitas \& Andrich 2013).

Upon this background, our goal was to study the daily interaction of birds with flowers and fruits of Acnistus arborescens in the Itatiaia National Park. In this sense, information was provided on the reproductive phenology of the plant species and also on behavior of bird visitors in order to infer on their potential role as pollinators/robbers and seed dispersers/predators.

\section{Material and Methods}

Study area

Itatiaia National Park is located in the Southwestern Rio de Janeiro and Southern Minas Gerais states $\left(22^{\circ} 15^{\prime}-22^{\circ} 30^{\prime} \mathrm{S}, 4^{\circ} 30^{\prime}-44^{\circ} 45^{\prime} \mathrm{W}\right)$ in Southeastern Brazil (Fig. 1). It covers an area of 28,084.3 hectares of Brazilian Atlantic Forest (Oliveira-Filho \& Fontes 2000) inserted in the Mantiqueira Mountain range. Vegetation at the elevation of this study is Montane Atlantic Forest (approx. 1,000 m a.s.l.). Climate is "Cfb" (Peel et al. 2007), with mild and rainy summers, interspersed with cold periods of low rainfall (Segadas-Vianna $\&$ Dau 1965). Annual precipitation averages 1,500 mm (Costa et al. 2012).

\section{Species studied}

Acnistus arborescens is a pioneer shrubtree species with fasciculate, pale-green, stem flowers, that measure about $3 \pm 0.2 \mathrm{~cm}$ (Mean \pm Standard deviation) long and $0.5 \pm 0.1 \mathrm{~cm}$ in diameter (Fig. 1), and exude a sweet and soft smell. Anthesis is diurnal, starting around $06: 00 \mathrm{~h}$, lasting approximately 12 hours. The fruit is a globose berry, measuring about $1.0 \pm 0.4 \mathrm{~cm}$ in diameter, with ca. 13\% of sugar (Verçoza et al. 2012). These fruits contain ca. 60 seeds (Athiê \& Dias 2012) and exhibit features associated with ornithochory, such as fleshy consistency, orange color, and absence of odor (Van der Pij1 1982).

Acnistus arborescens occurs from southern Mexico to Southern Brazil from sea level up to 2,000 m a.s.l. (Hunziker 2001). In Brazil, it has been recorded in several states of the Northeastern, Southeastern, and Southern regions (BFG 2018. In the Itatiaia National Park individuals of $A$. arborescens were found occupying only areas with forest edges, open areas and in degraded areas on natural recovery similar to that identified in other localities (Verçosa 2012; Athiê \& Dias 2012).

\section{Sampling procedures}

Eighteen individuals in reproductive condition were selected in INP. Phenological monitoring was conducted on a monthly basis between July 2015 and January 2016. The flowering (open flowers) and fruiting (mature and immature) phenophases were recorded considering the presence or absence of each phase. Flowering patterns were analyzed on individual and population levels, and described in terms of their duration, i.e., the number of months (Newstrom et al. 1994). The synchronization of the 
phenophases was evaluated based on the proportion of individuals presenting the same phenophase during a given period (Bencke \& Morellato 2002). Measurements of the fruits per flower (fruit set) were taken in all individuals. The largest and smallest distances between individuals were $1 \mathrm{~km}$ and $30 \mathrm{~m}$, respectively.

Visitors consuming of nectar and fruits were recorded between 6:00 and 18:00 h, during continuous sampling bouts of 180 minutes totalizing $54 \mathrm{~h}$ of observations equally distributed among the fourfocal individuals (Pizo \& Galetti 2010). During these observations, visitor type, number of individuals per species and duration of the visits, number of flowers and fruits foraged and consumption behavior were recorded. Birds were classified according to the fruit consumption method (sensu Parrini \& Pacheco 2011a): a) to macerate the pulp, b) to chew the fruits before ingesting them, or c) to swallow whole fruits, previously chewing them or not.

Foraging strategies for fruit consumption were classified as suggested by Pizo \& Galetti (2010): a) perched (when the bird remained on the plant while collecting and consuming the fruit), b) flying (when the bird arrived to the plant, collected the fruit and landed somewhere else to consume it), and c) on the ground (when birds ate fallen fruits lying on the ground).

Visit duration was standardized as suggested by Parrini \& Pacheco (2011a): a) short $(<3$ minutes), b) medium (3-10 minutes), and c) long ( $>10$ minutes). Subsequently, the total number of fruits eaten by each species was estimated as follows: (number of visits) $\times$ (number of fruits consumed per visit) $\times$ (length of each visit) $\times$ (number of individuals per visit), adapted from Aximoff \& Freitas (2009).

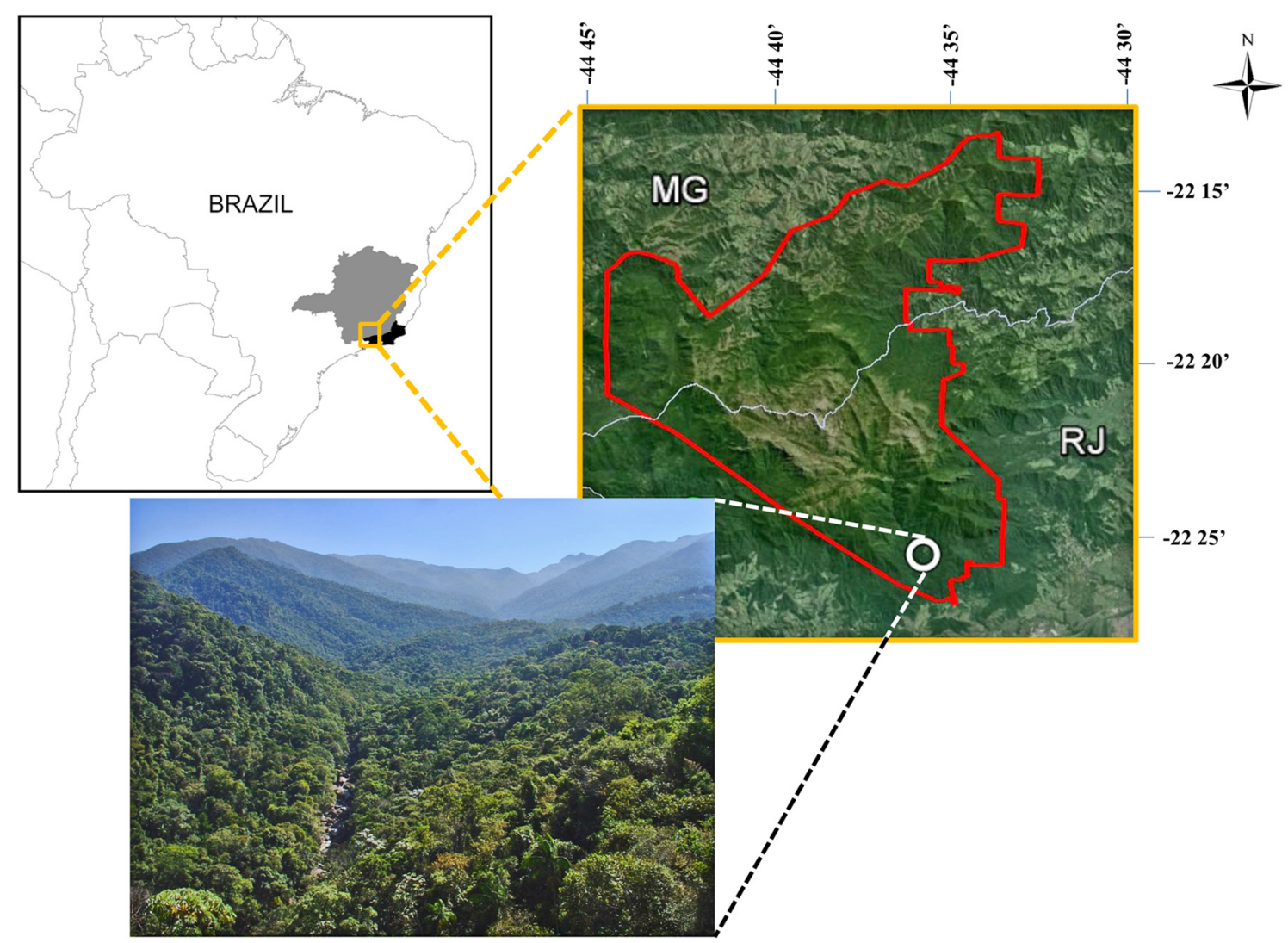

Figure 1 - a-c. Location of study site in the Itatiaia National Park - INP - a. in Southeastern Brazil, Minas Gerais state - MG (gray), Rio de Janeiro state - RJ (black) and the Itatiaia National Park (golden square); b. the location of the study area in the south part (white circle), inside limits of the INP (red line); c. the physiognomy of Brazilian Atlantic Forest in INP (left). 
Identification of birds was done in the field according to Ridgely et al. (2015). Additionally, photographs were sent to experts to confirm the identification. Classification and nomenclature of bird species follow the list of Brazilian Committee of Bird Ornithological Records (CBRO 2014). Endangered species were identified based on the official lists of the state of Rio de Janeiro (SEMA 1998) and on the national list (MMA 2014). Endemic species of Atlantic Forest were identified based in specific literature (Cracft 1985; Sick 1997; Silva et al. 2004; Bencke et al. 2006).

\section{Results}

\section{Phenology and plant features}

Flowering of Acnistus arborescens occurred between August and November with $2.6 \pm 0.6$ months per individual (Mean \pm Standard deviation), with a higher number of individuals flowering simultaneously in September and October (44.4\%). Fruiting occurred from September to December with $2.2 \pm 0.9$ months per individual, with a higher number of individuals bearing fruits in November $(38.8 \%)$. All plants had flowering and fruiting phenophases at the same time after the first month of flowering, but in December only fruiting was registered (Fig. 2). Fruit set was $87.3 \%$. The average number of flowers and fruits produced per individual was $3.024 \pm 32.4$ and $2.782 \pm 24.5$, respectively.

\section{Flower visitors}

Flowers were visited by seven species of hummingbirds, one bird and several bees, wasps, butterflies and flies. Flower visits started around 8:00 h, increased from 10:30 to $13: 00 \mathrm{~h}$ and then decreased, ending at 16:30 h. During the observation period nectar from 405 flowers was consumed. Most flower visits were made by hummingbirds being Phaethornis ruber the main

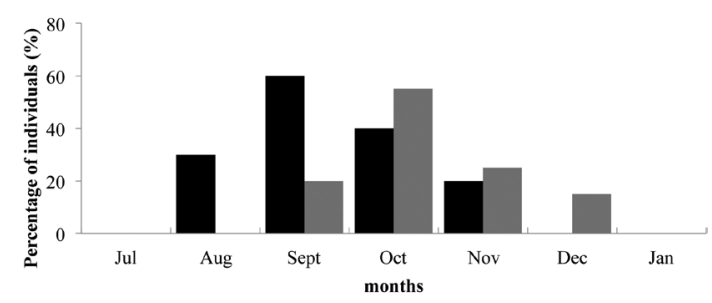

Figure 2 - Percentage of individuals $(\mathrm{n}=18)$ in flowering (black bars) and fruiting phases (gray bars) of Acnistus arborescens in Itatiaia National Park - INP. visitor with $32.1 \%$ of total visits (Tab. 1). Visits of all hummingbirds were concentrated during early morning until 10:00 (48\%) and mid to late afternoon (32\%), with some visits at different times throughout the morning and afternoon $(20 \%)$. During visits, hummingbird's beak contacts anthers and stigma, confirming their legitimate behavior and evidencing they can pollinate (Fig. 3 ). Only Coereba flaveola (Thraupidae) was observed visiting flowers as nectar thief. There were no records of agonistic interactions and only three species were recorded with more than one individual on the same plant (P. ruber, $L$. magnificus and A. lactea). Three hummingbird species are endemic to the Brazilian Atlantic Forest (Tab. 1).

\section{Frugivory}

We recorded 35 bird species consuming fruits of Acnistus arborescens, including Coereba flaveola (Tab. 2). Most visits were performed by members of the family Thraupidae with $53.9 \%$ of total. The species with the highest number of visits were Tangara desmaresti (10.9\%), Euphonia pectoralis $(7.7 \%)$, Tangara cayana $(7.3 \%)$ and $T$. cyanoventris (6.8\%) (Tab. 2). Most species (78\%) made short visits (Fig. 4).

All Psittacidae and three Thraupidae species (Tangara desmaresti, T. seledon and T. cayana) had the highest number of visits on intraspecific flocks of up to six individuals in the same plant. Mixed flocks with Tangara sayaca, T. cyanoventris, Ramphocelus bresilius and Cyanoloxia brissonii were identified. Intra- and interspecific agonistic interactions were rare, only in $10.5 \%$ of visits. Birds avoided confrontations changing places and exploring other branches, as observed with a group of Tangara seledon and between $T$. ornata and $T$. cyanoventris.

All Pipridae and almost all of Tyrannidae species ( $75 \%$ of total), were observed performing flights to collect fruits. In Turdidae, some species collected fruits perching on the branches and then flew. Most Thraupidae ( $80 \%$ of total) collected fruits while perching on branches. Only the Duskylegged Guan (P. obscura) was recorded feeding on fallen fruits on the ground, but also eating on the branches of $A$. arborescens. All the other species were recorded feeding perching on branches.

In most records, the consumption mode was chewing associated with macerating fruits. After collecting fruits, Psittacidae, Thraupidae, and Fringilidae chewed and macerated them. All 
Table 1 - Nectarivorous bird species and behavior in flowers of Acnistus arborescens (Solanaceae) in Itatiaia National Park. AF $=$ Atlantic Forest; $\mathrm{N}^{\mathrm{o}}=$ number.

\begin{tabular}{|c|c|c|c|c|c|c|c|c|}
\hline \multirow{2}{*}{$\begin{array}{l}\text { Order, Family } \\
\text { Species }\end{array}$} & \multirow[b]{2}{*}{ Common name } & \multirow{2}{*}{$\begin{array}{c}\text { AF } \\
\text { Endemic }\end{array}$} & \multicolumn{3}{|c|}{ Visits } & \multicolumn{3}{|c|}{ Visited flowers } \\
\hline & & & $\mathbf{N}^{\mathbf{o}}$ & Minutes & $\begin{array}{c}\mathrm{N}^{\circ} \text { of } \\
\text { individuals }\end{array}$ & $\begin{array}{l}\text { Average } \\
\text { per visit }\end{array}$ & $\begin{array}{l}\text { Legitimate } \\
\qquad(\%)\end{array}$ & $\begin{array}{l}\text { Illegitimate } \\
(\%)\end{array}$ \\
\hline \multicolumn{9}{|l|}{$\begin{array}{l}\text { Apodiformes, } \\
\text { Trochilidae }\end{array}$} \\
\hline $\begin{array}{l}\text { Phaethornis ruber } \\
\text { (Linnaeus 1758) }\end{array}$ & Reddish Hermit & - & 12 & $5.7 \pm 0.9$ & $1.9 \pm 1.1$ & 10.8 & $130(39.3)$ & - \\
\hline $\begin{array}{l}\text { Lophornis magnificus } \\
\text { (Vieillot 1817) }\end{array}$ & Frilled Coquette & - & 6 & $2.3 \pm 1.6$ & $1.4 \pm 0.7$ & 7.2 & $47(15.0)$ & - \\
\hline $\begin{array}{l}\text { Thalurania glaucopis } \\
\text { (Gmelin 1788) }\end{array}$ & $\begin{array}{l}\text { Violet-capped } \\
\text { Woodnymph }\end{array}$ & $\mathrm{X}$ & 7 & $2.5 \pm 1.2$ & $1.0 \pm 0$ & 6.6 & $38(12.0)$ & - \\
\hline $\begin{array}{l}\text { Amazilia versicolor } \\
\text { (Vieillot 1818) }\end{array}$ & $\begin{array}{l}\text { Versicolored } \\
\text { Emerald }\end{array}$ & - & 3 & $1.5 \pm 0.6$ & $1.0 \pm 0$ & 2.7 & $5(1.4)$ & - \\
\hline $\begin{array}{l}\text { Amazilia fimbriata } \\
\text { (Gmelin 1788) }\end{array}$ & $\begin{array}{l}\text { Glittering-throated } \\
\text { Emerald }\end{array}$ & - & 4 & $1.2 \pm 0.8$ & $1.0 \pm 0$ & 3.4 & $14(4.0)$ & - \\
\hline $\begin{array}{l}\text { Amazilia lactea } \\
\text { (Lesson 1832) }\end{array}$ & $\begin{array}{l}\text { Sapphire-spangled } \\
\text { Emerald }\end{array}$ & $\mathrm{X}$ & 10 & $3.3 \pm 1.9$ & $1.5 \pm 0.6$ & 7.4 & $74(22.4)$ & - \\
\hline $\begin{array}{l}\text { Leucochloris albicollis } \\
\text { (Vieillot 1818) }\end{array}$ & $\begin{array}{l}\text { White-throated } \\
\text { Hummingbird }\end{array}$ & $X$ & 4 & $1.3 \pm 0.5$ & $1.0 \pm 0$ & 4.8 & $19(5.8)$ & - \\
\hline \multicolumn{9}{|l|}{$\begin{array}{l}\text { Passeriformes, } \\
\text { Thraupidae }\end{array}$} \\
\hline $\begin{array}{l}\text { Coereba flaveola } \\
\text { (Linnaeus 1758)* }\end{array}$ & Bananaquit & - & 8 & $2.2 \pm 0.2$ & $1.0 \pm 0$ & 9.2 & - & $78(100)$ \\
\hline Total & & & 54 & - & - & - & 327 & 78 \\
\hline
\end{tabular}

Psittacidae species, as well as some Traupidae species (T. desmaresti, T. sayaca, T. palmarum and $T$. ornata), were additionally observed consuming green fruits (Fig. 5). Of the 3,388 fruits accounted for the observations, $52.3 \%$ were consumed by Thraupidae species. Other families with representative values were Turdidae (16\%), Fringilidae (9\%) and Psittacidae (6\%). The most frequent fruit consumers were: $T$. desmaresti (11.8\%), Turdus rufiventris (10.3\%) and E. pectoralis $(8.9 \%)$.

Sixteen bird species recorded consuming fruits are considered endemic to the Brazilian Atlantic Forest (Tab. 1). Cyanoloxia brissonii is threatened in Rio de Janeiro State (i.e. Vulnerable, SEMA 1998).

\section{Discussion}

Acnistus arborescens is an important food source for bird species in Itatiaia National Park. The
42 bird species observed feeding on floral nectar and fruits represents nearly $10 \%$ of total bird species recorded in the park (ICMBio 2013). Besides that, richness of birds consuming nectar of $A$. arborescens at INP ( 8 species) was higher than that reported in previous studies. In the Pedra Branca State Park, a stretch of Atlantic forest located in western city of Rio de Janeiro, only two hummingbird species were recorded (Verçosa et al. 2012). In Ecuador and Peru, $A$. arborescens was visited exclusively by insects while hummingbird species visited other plant species (Smith et al. 2008).

The largest number of hummingbird species in INP, than those recorded by Smith et al. (2008), may allow that some of these animals act as specific visitors of $A$. arborescens. Only two of the seven hummingbird species observed feeding on $A$. arborescens were also detected as nectar consumers in the assemblage of 40 ornithophilous plant species at Itatiaia National Park (Canela 

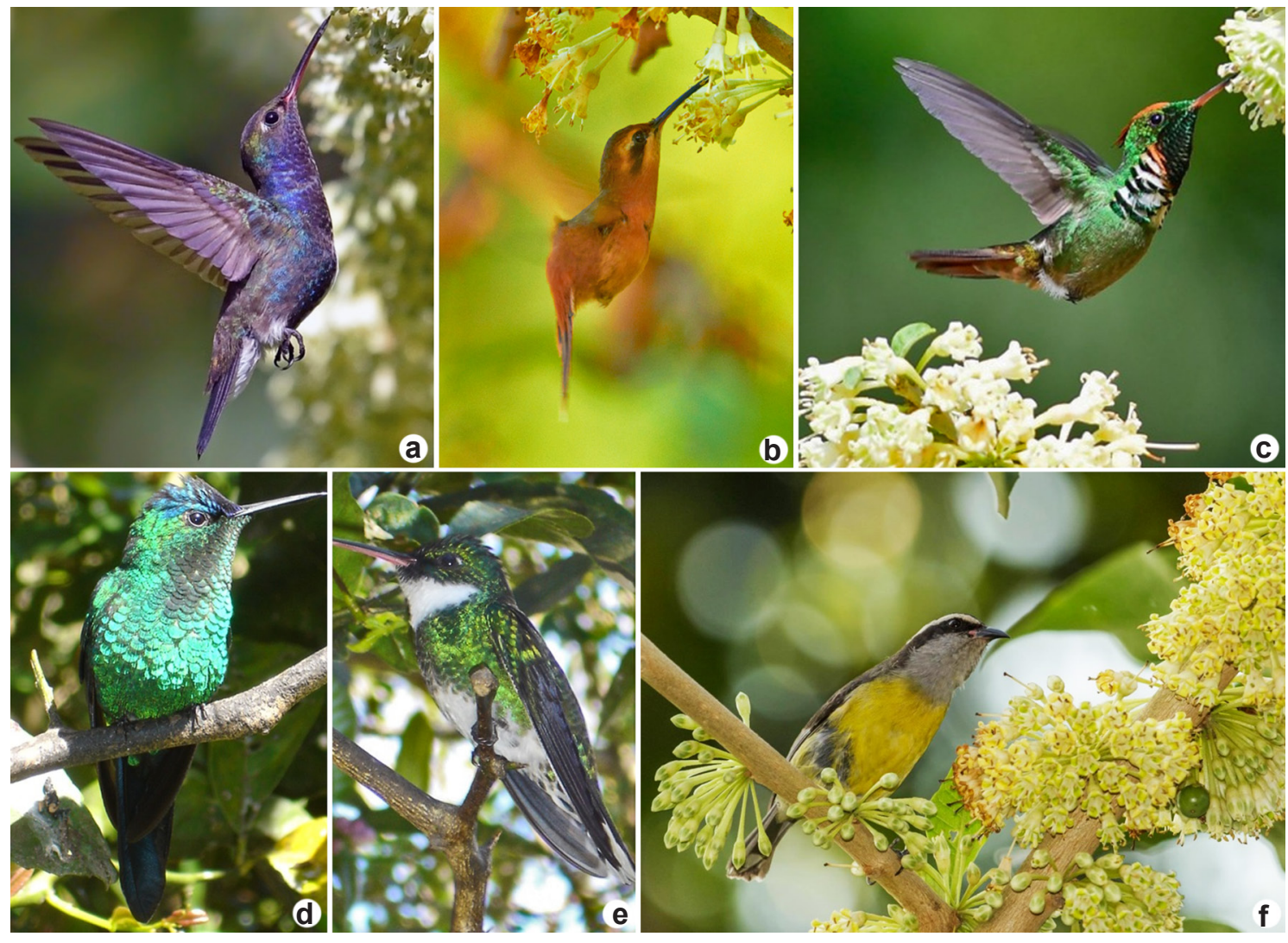

Figure 3 - a-f. Some of the nectarivorous bird species in flowers of Acnistus arborescens in Itatiaia National Park - a. Amazilia lactea; b. Phaethornis ruber; c. Lophornis magnificus; d. Thalurania glaucopis; e. Leucochloris albicollis Trochilidae; f. Coereba flaveola Thraupidae.

2006; Aximoff \& Freitas 2009; Wolowski et al. 2013a). The other five hummingbird species act almost exclusively at $A$. arborescens during their flowering. This may be related to the fact of hundreds of open flowers available in $A$. arborescens compensate the small amount of nectar available in each one (Smith et al. 2008). In this sense, it reinforces the local importance of $A$. arborescens in providing resources for the maintenance of some hummingbird species.

Richness of birds consuming fruits of $A$. arborescens at INP (35 species) was also higher than that reported in previous studies: 21 species in Costa Rica (Wheelwrigh et al. 1984), 16 species in Jamaica, (Cruz 1981), and in Brazil, 13 species (Verçoza et al. 2012) and 15 species (Athiê \& Dias 2012). In the Brazilian Atlantic Rain Forest, some plant species presented high richness of fruit consuming birds: 32 in Alchornea triplinervia (Euphorbiaceae) (Parrini \& Pacheco 2011a), 30 in Cupania oblongifolia (Sapindaceae) (Parrini \& Pacheco 2014), and 63 species in Rapanea schwackeana (Myrsinaceae) (Pineschi 1990). The high richness of consuming fruit birds in INP, including endangered species, can be directly related to the more than 400 bird species already recorded in this protected area (ICMBio 2013).

Some features of $A$. arborescens may be considered as effective to attract more consumers and potential dispersers (Athiê \& Dias 2012; Verçosa et al. 2012). One of them would be the high proportion of fruit pulp during the rainy season (Morellato \& Leitão Filho 1992; Pereira \& Mantovani 2007; Parrini \& Pacheco 2011a,b, 2014). The greater resources supply in the rainy season may be identified as the main factor responsible for the seasonal movements of birds to these places (Galetti et al. 2000; Morellato et al. 2000; Malizia 2001). Indded, a greater number of bird species was identified in the rainy season $(\mathrm{n}=68)$ than in the dry season $(\mathrm{n}=42)$ in specific locality in Itatiaia National Park (Gouvea et al. 2005). Another important feature would be the high amount of fruits produced and their extended period of exposure to 
Table 2 - Frugivorous bird species and behavior in fruits of Acnistus arborescens (Solanaceae) in Itatiaia National Park. AF $=$ Atlantic Forest; $\mathrm{N}^{\mathrm{o}}=$ number.

\begin{tabular}{|c|c|c|c|c|c|c|c|c|c|}
\hline \multirow{2}{*}{$\begin{array}{l}\text { Order, Family } \\
\text { Species }\end{array}$} & \multirow[b]{2}{*}{ Common name } & \multirow[b]{2}{*}{$\begin{array}{c}\mathrm{AF} \\
\text { Endemic }\end{array}$} & \multicolumn{3}{|c|}{ Visits } & \multicolumn{4}{|c|}{ Fruit Consumption } \\
\hline & & & $\mathbf{N}^{\mathbf{o}}$ & Minutes & Mode & Method & $\begin{array}{l}\text { Average } \\
\text { per visit }\end{array}$ & Strategy & $\begin{array}{l}\text { Total } \\
(\%)\end{array}$ \\
\hline \multicolumn{10}{|l|}{$\begin{array}{l}\text { Galliformes, } \\
\text { Cracidae }\end{array}$} \\
\hline $\begin{array}{l}\text { Penelope obscura } \\
\text { Temminck } 1815\end{array}$ & $\begin{array}{l}\text { Dusky-legged } \\
\text { Guan }\end{array}$ & $\mathrm{X}$ & 6 & $0.7 \pm 0.3$ & $\mathrm{~S}$ & $\mathrm{P}, \mathrm{G}$ & 15.8 & SW & $95(2.8)$ \\
\hline \multicolumn{10}{|l|}{$\begin{array}{l}\text { Columbiformes, } \\
\text { Columbidae }\end{array}$} \\
\hline $\begin{array}{l}\text { Patagioenas picazuro } \\
\text { (Temminck 1813) }\end{array}$ & Picazuro Pigeon & - & 5 & $0.6 \pm 0.2$ & $\mathrm{~S}$ & $\mathrm{P}$ & 17.4 & SW & $87(2.6)$ \\
\hline \multicolumn{10}{|l|}{$\begin{array}{l}\text { Psittaciformes, } \\
\text { Psittacidae }\end{array}$} \\
\hline $\begin{array}{l}\text { Pyrrhura frontalis } \\
\text { (Vieillot 1817) }\end{array}$ & $\begin{array}{l}\text { Maroon-bellied } \\
\text { Parakeet }\end{array}$ & $\mathrm{X}$ & 18 & $2.2 \pm 0.7$ & B & $\mathrm{P}$ & 4.4 & $\mathrm{CH}, \mathrm{MC}$ & $79(2.3)$ \\
\hline $\begin{array}{l}\text { Forpus xanthopterygius } \\
\text { (Spix 1824) }\end{array}$ & $\begin{array}{l}\text { Blue-winged } \\
\text { Parrotlet }\end{array}$ & - & 12 & $1.5 \pm 0.8$ & B & $\mathrm{P}$ & 5.4 & $\mathrm{CH}, \mathrm{MC}$ & $65(1.9)$ \\
\hline $\begin{array}{l}\text { Brotogeris tirica } \\
\text { (Gmelin 1788) }\end{array}$ & Plain Parakeet & $\mathrm{X}$ & 9 & $1.1 \pm 0.9$ & B & $\mathrm{P}$ & 5.8 & $\mathrm{CH}, \mathrm{MC}$ & $52(1.5)$ \\
\hline \multicolumn{10}{|l|}{$\begin{array}{l}\text { Passeriformes, } \\
\text { Pipridae }\end{array}$} \\
\hline $\begin{array}{l}\text { Manacus manacus } \\
\text { (Linnaeus 1766) }\end{array}$ & $\begin{array}{l}\text { White-bearded } \\
\text { Manakin }\end{array}$ & - & 7 & $0.9 \pm 0.5$ & $\mathrm{~S}$ & $\mathrm{~V}, \mathrm{P}$ & 3 & $\mathrm{MC}$ & $21(0.6)$ \\
\hline $\begin{array}{l}\text { Chiroxiphia caudata } \\
\text { (Shaw \& Nodder 1793) }\end{array}$ & $\begin{array}{l}\text { Swallow-tailed } \\
\text { Manakin }\end{array}$ & $\mathrm{X}$ & 8 & $1.0 \pm 0.2$ & $\mathrm{~S}$ & $\mathrm{~V}, \mathrm{P}$ & 2.8 & $\mathrm{MC}$ & $22(0.6)$ \\
\hline \multicolumn{10}{|l|}{$\begin{array}{l}\text { Passeriformes, } \\
\text { Tyrannidae }\end{array}$} \\
\hline $\begin{array}{l}\text { Elaenia flavogaster } \\
\text { (Thunberg 1822) }\end{array}$ & $\begin{array}{l}\text { Yellow-bellied } \\
\text { Elaenia }\end{array}$ & - & 8 & $1.0 \pm 0.3$ & $\mathrm{~S}$ & $\mathrm{P}$ & 2.4 & $\mathrm{MC}$ & $19(0.6)$ \\
\hline $\begin{array}{l}\text { Pitangus sulphuratus } \\
\text { (Linnaeus 1766) }\end{array}$ & Great Kiskadee & - & 34 & $4.2 \pm 1.1$ & $\mathrm{~S}$ & $\mathrm{~V}, \mathrm{P}$ & 0.7 & $\mathrm{MC}$ & $23(0.7)$ \\
\hline $\begin{array}{l}\text { Megarynchus pitangua } \\
\text { (Linnaeus 1766) }\end{array}$ & $\begin{array}{l}\text { Boat-billed } \\
\text { Flycatcher }\end{array}$ & - & 13 & $1.6 \pm 0.5$ & $\mathrm{~S}$ & $\mathrm{~V}, \mathrm{P}$ & 4.2 & $\mathrm{MC}$ & $54(1.6)$ \\
\hline $\begin{array}{l}\text { Myiozetetes similis } \\
\text { (Spix 1825) }\end{array}$ & Social Flycatcher & - & 11 & $1.4 \pm 0.4$ & $\mathrm{~S}$ & $\mathrm{~V}, \mathrm{P}$ & 3.9 & $\mathrm{MC}$ & $43(1.3)$ \\
\hline \multicolumn{10}{|l|}{$\begin{array}{l}\text { Passeriformes, } \\
\text { Turdidae }\end{array}$} \\
\hline $\begin{array}{l}\text { Turdus flavipes } \\
\text { (Vieillot 1818) }\end{array}$ & $\begin{array}{l}\text { Yellow-legged } \\
\text { Thrush }\end{array}$ & - & 12 & $1.5 \pm 0.3$ & S & $\mathrm{V}, \mathrm{P}$ & 2.6 & $\mathrm{MN}$ & $31(0.9)$ \\
\hline $\begin{array}{l}\text { Turdus leucomelas } \\
\text { (Vieillot 1818) }\end{array}$ & $\begin{array}{l}\text { Pale-breasted } \\
\text { Thrush }\end{array}$ & - & 31 & $3.8 \pm 1.1$ & S & $\mathrm{P}$ & 4.3 & $\mathrm{MN}$ & $\begin{array}{c}133 \\
(3.9)\end{array}$ \\
\hline $\begin{array}{l}\text { Turdus rufiventris } \\
\text { (Vieillot 1818) }\end{array}$ & $\begin{array}{l}\text { Rufous-bellied } \\
\text { Thrush }\end{array}$ & - & 37 & $4.6 \pm 1.4$ & S & $\mathrm{V}, \mathrm{P}$ & 9.5 & MN & $\begin{array}{c}350 \\
(10.3)\end{array}$ \\
\hline
\end{tabular}




\begin{tabular}{|c|c|c|c|c|c|c|c|c|c|}
\hline \multirow[b]{2}{*}{$\begin{array}{l}\text { Order, Family } \\
\text { Species }\end{array}$} & \multirow[b]{2}{*}{ Common name } & \multirow[b]{2}{*}{$\begin{array}{c}\mathbf{A F} \\
\text { Endemic }\end{array}$} & \multicolumn{3}{|c|}{ Visits } & \multicolumn{4}{|c|}{ Fruit Consumption } \\
\hline & & & $\mathbf{N}^{0}$ & Minutes & Mode & Method & $\begin{array}{l}\text { Average } \\
\text { per visit }\end{array}$ & Strategy & $\begin{array}{l}\text { Total } \\
(\%)\end{array}$ \\
\hline $\begin{array}{l}\text { Turdus amaurochalinus } \\
\text { (Cabanis 1850) }\end{array}$ & $\begin{array}{l}\text { Creamy-bellied } \\
\text { Thrush }\end{array}$ & - & 18 & $2.2 \pm 0.7$ & $\mathrm{~S}$ & $\mathrm{P}$ & 2.4 & MN & $43(1.3)$ \\
\hline \multicolumn{10}{|l|}{$\begin{array}{l}\text { Passeriformes, } \\
\text { Mimidae }\end{array}$} \\
\hline $\begin{array}{l}\text { Mimus saturninus } \\
\text { (Lichtenstein 1823) }\end{array}$ & $\begin{array}{l}\text { Chalk-browed } \\
\text { Mockingbird }\end{array}$ & - & 8 & $1.0 \pm 0.1$ & S & $\mathrm{P}$ & 4.1 & SW & $33(1.0)$ \\
\hline \multicolumn{10}{|l|}{$\begin{array}{l}\text { Passeriformes, } \\
\text { Passerellidae }\end{array}$} \\
\hline $\begin{array}{l}\text { Zonotrichia capensis } \\
\text { (Statius Muller 1776) }\end{array}$ & $\begin{array}{l}\text { Rufous-collared } \\
\text { Sparrow }\end{array}$ & - & 13 & $1.6 \pm 0.2$ & $\mathrm{~S}$ & $\mathrm{P}$ & 3.5 & SW & $45(1.3)$ \\
\hline $\begin{array}{l}\text { Arremon semitorquatus } \\
\text { (Swainson 1838) }\end{array}$ & $\begin{array}{l}\text { Half-collared } \\
\text { Sparrow }\end{array}$ & $X$ & 12 & $1.5 \pm 0.4$ & S & $\mathrm{P}$ & 4.6 & SW & $55(1.6)$ \\
\hline \multicolumn{10}{|l|}{$\begin{array}{l}\text { Passeriformes, } \\
\text { Thraupidae }\end{array}$} \\
\hline $\begin{array}{l}\text { Coereba flaveola } \\
\text { (Linnaeus 1758)* }\end{array}$ & Bananaquit & - & 22 & $2.7 \pm 0.3$ & $\mathrm{~S}, \mathrm{M}$ & $\mathrm{V}, \mathrm{P}$ & 3 & $\mathrm{CH}, \mathrm{MC}$ & $67(2.0)$ \\
\hline $\begin{array}{l}\text { Saltator similis } \\
\text { (d'Orbigny \& } \\
\text { Lafresnaye 1837) }\end{array}$ & $\begin{array}{l}\text { Green-winged } \\
\text { Saltator }\end{array}$ & - & 21 & $2.6 \pm 0.7$ & $\mathrm{~S}$ & $\mathrm{P}$ & 2.1 & $\mathrm{CH}, \mathrm{MC}$ & $45(1.3)$ \\
\hline $\begin{array}{l}\text { Thlypopsis sordida } \\
\text { (d'Orbigny \& } \\
\text { Lafresnaye 1837) }\end{array}$ & $\begin{array}{l}\text { Orange-headed } \\
\text { Tanager }\end{array}$ & - & 12 & $1.5 \pm 0.2$ & $\mathrm{~S}$ & $\mathrm{P}$ & 2.7 & $\mathrm{CH}, \mathrm{MC}$ & $32(0.9)$ \\
\hline $\begin{array}{l}\text { Tachyphonus coronatus } \\
\text { (Vieillot 1822) }\end{array}$ & $\begin{array}{l}\text { Ruby-crowned } \\
\text { Tanager }\end{array}$ & $\mathrm{X}$ & 46 & $5.7 \pm 0.5$ & $\mathrm{~S}$ & $\mathrm{P}$ & 2.1 & $\mathrm{CH}, \mathrm{MC}$ & $98(2.9)$ \\
\hline $\begin{array}{l}\text { Ramphocelus bresilius } \\
\text { (Linnaeus 1766) }\end{array}$ & Brazilian Tanager & $X$ & 20 & $2.5 \pm 0.9$ & $\mathrm{~S}, \mathrm{M}$ & $\mathrm{P}$ & 2.3 & $\mathrm{CH}, \mathrm{MC}$ & $45(1.3)$ \\
\hline $\begin{array}{l}\text { Tangara seledon } \\
\text { (Muller 1776) }\end{array}$ & $\begin{array}{l}\text { Green-headed } \\
\text { Tanager }\end{array}$ & $X$ & 18 & $2.2 \pm 0.4$ & B & $\mathrm{P}$ & 3.7 & $\mathrm{CH}, \mathrm{MC}$ & $67(2.0)$ \\
\hline $\begin{array}{l}\text { Tangara cyanoventris } \\
\text { (Vieillot 1819) }\end{array}$ & $\begin{array}{l}\text { Gilt-edged } \\
\text { Tanager }\end{array}$ & $X$ & 55 & $6.8 \pm 0.9$ & $\begin{array}{l}\mathrm{S}, \mathrm{B} \\
\mathrm{M}\end{array}$ & $\mathrm{P}$ & 4.5 & $\mathrm{CH}, \mathrm{MC}$ & $\begin{array}{l}250 \\
(7.4)\end{array}$ \\
\hline $\begin{array}{l}\text { Tangara desmaresti } \\
\text { (Vieillot 1819) }\end{array}$ & $\begin{array}{l}\text { Brassy-breasted } \\
\text { Tanager }\end{array}$ & $X$ & 88 & $\begin{array}{c}10.9 \pm \\
2.3\end{array}$ & B & $\mathrm{V}, \mathrm{P}$ & 4.5 & $\mathrm{CH}, \mathrm{MC}$ & $\begin{array}{c}400 \\
(11.8)\end{array}$ \\
\hline $\begin{array}{l}\text { Tangara sayaca } \\
\text { (Linnaeus 1766) }\end{array}$ & Sayaca Tanager & - & 39 & $4.8 \pm 1.7$ & $\mathrm{~S}, \mathrm{M}$ & $\mathrm{P}$ & 5 & $\mathrm{CH}, \mathrm{MC}$ & $\begin{array}{c}194 \\
(5.7)\end{array}$ \\
\hline $\begin{array}{l}\text { Tangara palmarum } \\
\text { (Wied 1823) }\end{array}$ & Palm Tanager & - & 12 & $1.5 \pm 0.2$ & S & $\mathrm{P}$ & 1.9 & $\mathrm{CH}, \mathrm{MC}$ & $23(0.7)$ \\
\hline $\begin{array}{l}\text { Tangara ornata } \\
\text { (Sparrman 1789) }\end{array}$ & $\begin{array}{l}\text { Golden- } \\
\text { chevroned } \\
\text { Tanager }\end{array}$ & $X$ & 37 & $4.6 \pm 0.7$ & $\mathrm{~S}$ & $\mathrm{P}$ & 4.3 & $\mathrm{CH}, \mathrm{MC}$ & $\begin{array}{l}158 \\
(4.7)\end{array}$ \\
\hline $\begin{array}{l}\text { Tangara cayana } \\
\text { (Linnaeus 1776) }\end{array}$ & $\begin{array}{l}\text { Burnished-buff } \\
\text { Tanager }\end{array}$ & - & 59 & $7.3 \pm 3.7$ & B & $\mathrm{P}$ & 4.2 & $\mathrm{CH}, \mathrm{MC}$ & $\begin{array}{l}250 \\
(7.4)\end{array}$ \\
\hline $\begin{array}{l}\text { Cissopis leverianus } \\
\text { (Gmelin 1788) }\end{array}$ & Magpie Tanager & - & 8 & $1.0 \pm 0.5$ & S & $\mathrm{P}$ & 2.6 & $\mathrm{CH}, \mathrm{MC}$ & $21(0.6)$ \\
\hline
\end{tabular}




\begin{tabular}{|c|c|c|c|c|c|c|c|c|c|}
\hline \multirow[b]{2}{*}{$\begin{array}{l}\text { Order, Family } \\
\text { Species }\end{array}$} & \multirow[b]{2}{*}{ Common name } & \multirow[b]{2}{*}{$\begin{array}{c}\text { AF } \\
\text { Endemic }\end{array}$} & \multicolumn{3}{|c|}{ Visits } & \multicolumn{4}{|c|}{ Fruit Consumption } \\
\hline & & & $\mathbf{N}^{0}$ & Minutes & Mode & Method & $\begin{array}{l}\text { Average } \\
\text { per visit }\end{array}$ & Strategy & $\begin{array}{l}\text { Total } \\
(\%)\end{array}$ \\
\hline $\begin{array}{l}\text { Dacnis cayana } \\
\text { (Linnaeus } 1766 \text { ) }\end{array}$ & Blue Dacnis & - & 19 & $2.4 \pm 0.9$ & $\mathrm{~S}$ & $\mathrm{~V}, \mathrm{P}$ & 2.4 & $\mathrm{CH}, \mathrm{MC}$ & $45(1.3)$ \\
\hline $\begin{array}{l}\text { Hemithraupis } \\
\text { ruficapilla } \\
\text { (Vieillot 1818) }\end{array}$ & $\begin{array}{l}\text { Rufous-headed } \\
\text { Tanager }\end{array}$ & $\mathrm{X}$ & 19 & $2.4 \pm 0.7$ & $\mathrm{~S}$ & $\mathrm{P}$ & 4.1 & $\mathrm{CH}, \mathrm{MC}$ & $78(2.3)$ \\
\hline \multicolumn{10}{|l|}{$\begin{array}{l}\text { Passeriformes, } \\
\text { Cardinalidae }\end{array}$} \\
\hline $\begin{array}{l}\text { Cyanoloxia brissonii } \\
\text { (Lichtenstein 1823) }\end{array}$ & $\begin{array}{l}\text { Ultramarine } \\
\text { Grosbeak }\end{array}$ & - & 8 & $1.0 \pm 0.3$ & $\mathrm{~S}, \mathrm{M}$ & $\mathrm{P}$ & 8.1 & $\mathrm{CH}$ & $65(1.9)$ \\
\hline \multicolumn{10}{|l|}{$\begin{array}{l}\text { Passeriformes, } \\
\text { Fringillidae }\end{array}$} \\
\hline $\begin{array}{l}\text { Euphonia pectoralis } \\
\text { (Latham 1801) }\end{array}$ & $\begin{array}{l}\text { Chestnut-bellied } \\
\text { Euphonia }\end{array}$ & $\mathrm{X}$ & 62 & $7.7 \pm 2.5$ & $\mathrm{~S}$ & $\mathrm{P}$ & 4.8 & $\mathrm{CH}, \mathrm{MC}$ & $\begin{array}{l}301 \\
(8.9)\end{array}$ \\
\hline Total & & - & 807 & - & - & - & - & - & 3389 \\
\hline
\end{tabular}

consumers (Cruz 1981; Wheelwrigh et al. 1984; Levey 1987; Verçoza et al. 2012). Its annual reproductive phenology ( 5 months) promotes the supply of important resources for foraging of the birds richness of Itatiaia National Park.

Thraupidae, Turdidae and Tyrannidae were the main dispersers of $A$. arborescens (78\%) in Itatiaia. These families were also present among those with higher number of species in studies of A. arborescens in Jamaica (Cruz 1981), Costa Rica (Wheelwrigh et al. 1984) and Brazil (Athiê \& Dias 2012; Verçoza et al. 2012). Other plant species of the Atlantic Rain Forest, as Cupania oblongifolia (Sapindaceae) (Parrini \& Pacheco 2014) and Miconia spp. (Melastomataceae) (Parrini \& Pacheco 2011b) were also dispersed by these bird families. Thraupidae species accounted for more than half of fruit consumers, a close percentage recorded for shrub-tree species of the Atlantic Rain Forest of southeastern Brazil (Manhães et al. 2003; Parrini et al. 2008; Parrini \& Pacheco 2011a,b, 2014). Tyranidae (26 species) and Thraupidae (21 species) were the families with the highest number of species recorded as dispersing species of Rapanea (Myrsinaceae) in the Itatiaia National Park (Pineschi 1990). Although we have not evaluated the seed dispersal efficiency, this is directilly related to feeding behavior and with the different ways of fruit consumption of $A$. arborescens (Cruz 1981).
Habitat characteristics where A. arborescens grows, such as clearings, secondary forests and edges of trails and roads, can also encourage visitation by bird species that use open and disturbed areas (Galetti et al. 2003; Athiê \& Dias 2012), as other typical forest areas (Fadini \& Marco Jr. 2004; Jordano et al. 2006). This can also facilitate the arrival of seeds brought by birds from adjacent conservation areas. The importance of birds species tipically found in forest interior that are attracted to fruting plants at the forest borders could transport seeds from plants located in forest interior to forest borders, and potentially increase plant diversity in this disturbed areas has to be highlighed (Pizzo 2007; Campos et al. 2012). The use of resources by Chiroxiphia caudata (obligatory frugivore) varied according to the availability of fruits and the conservation status (structural complexity) of forests (Hasui et al. 2009).

Birds are preferentially used to evaluate the effectiveness of restored areas because of their mobility, the speed at which they colonize new environments, their ability to connect habitats through seed dispersal, and their maintenance of gene flow among plant populations (Campos et al. 2012; Cavallero et al. 2013; Vogel et al. 2015). In Brazil, the main species of birds related to the process of forest regeneration are those that are more generalist, with a diet of fruits and insects and that live on forest edges and open areas (Pizo 

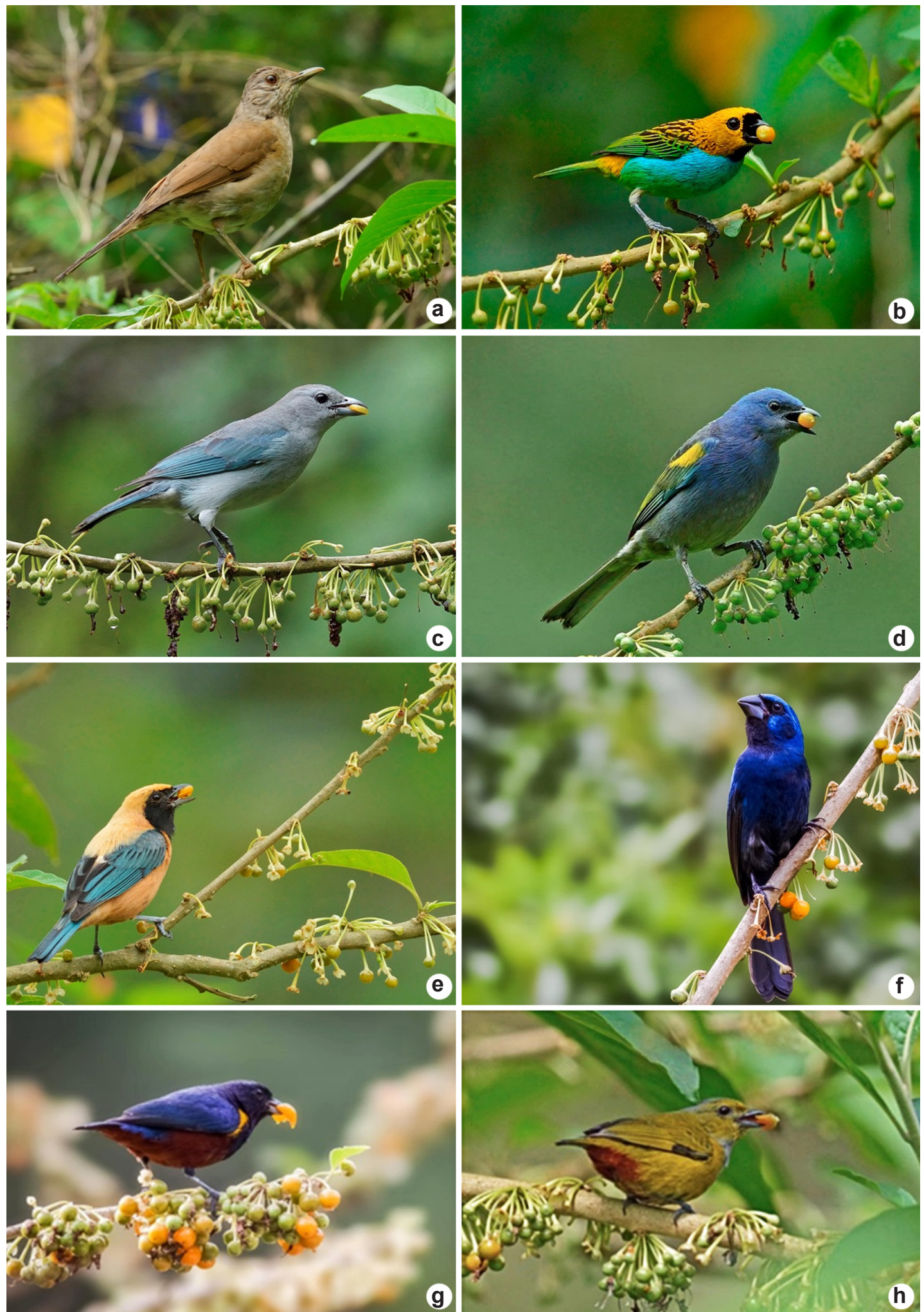

Figure 4 - a-h. Some of the main frugivorous birds of Acnistus arborescens in Itatiaia National Park - a. Turdus leucomelas Turdidae; b. Tangara cyanoventris; c. T. sayaca; d. T. ornata; e. T. cayana Thraupidae; f. Cyanoloxia brissonii (threatened) Cardinalidae; g. Euphonia pectoralis - male; h. Euphonia pectoralis - female Fringillidae. 
2007). These species belong mainly to the families Tyrannidae, Turdidae and Thraupidae (Campos et al. 2012). In addition, the birds of these families present great potential as dispersers, since they generally feed on the fruits without destroying the seeds (Athie \& Dias 2012).

The Ultramarine Grosbeak Cyanoloxia brissonii was the only endangered species feeding on A. arborescens. This bird is territorialist, and if there is a couple in a certain location, it will only be possible to find another couple at a distance, and if one male invades the territory of another, there will surely be a conflict, and it will be very violent (Sick 1997). In 20 years (1992 to 2012) this was the species with the highest number of seizures (more than 4,000 individuals) by the environmental police in one of the largest cities in Brazil (i.e. Belo Horizonte in the state of Minas Gerais) (Souza \& Vilela 2013).
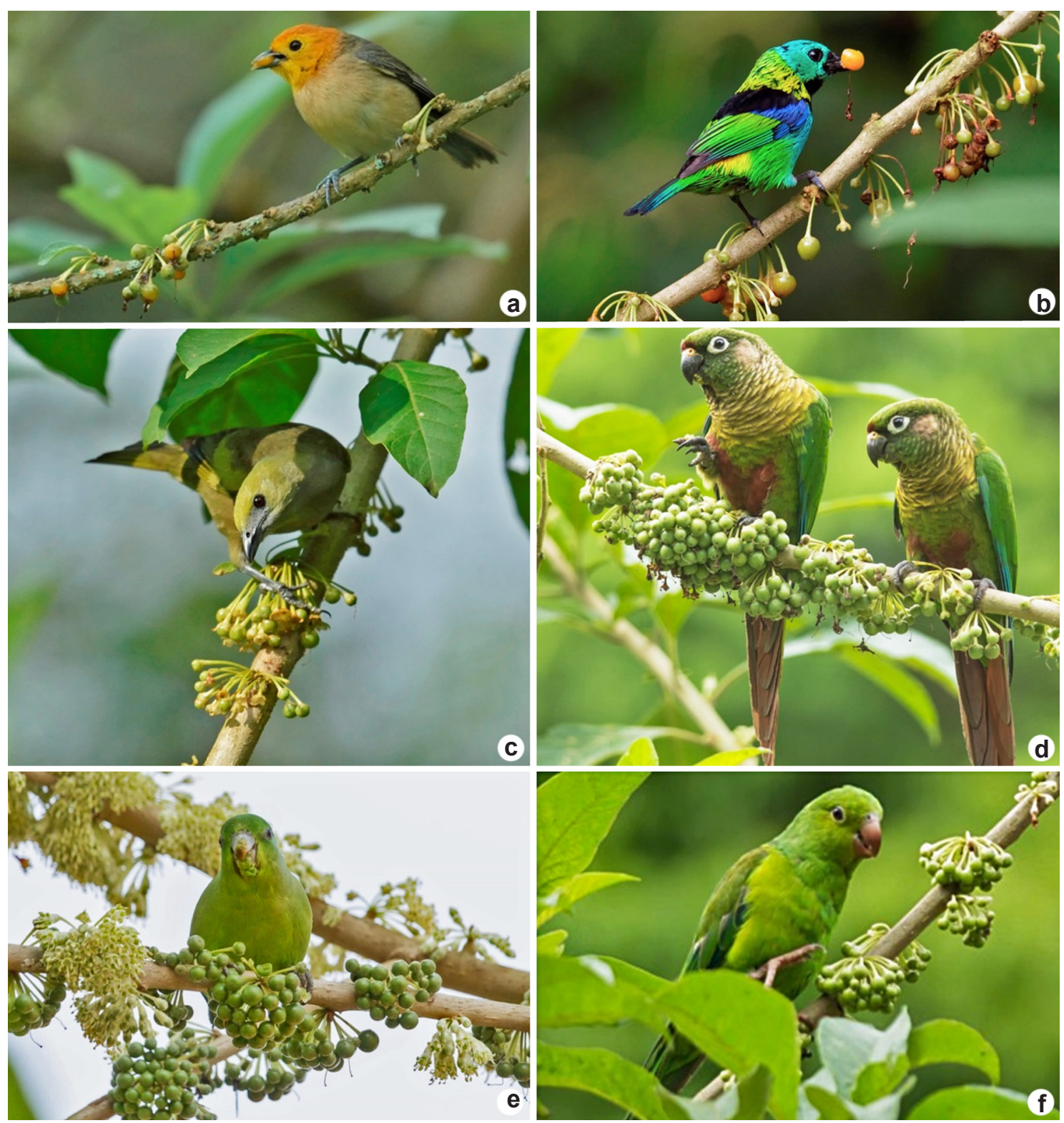

Figure 5 - a-e. Some of the green fruits consumers of Acnistus arborescens and that also had the highest number of visits on intraspecific flocks in the same plant - a. Tangara desmaresti; b. T. seledon; c. T. palmarum (Thraupidae); d. Pyrrhura frontalis, Brotogeris tirica; e. Forpus xanthopterygius (Psittacidae). 
Considering that Acnistus arborescens can support a wide assenblage of nectarivorous and frugivorous birds, this can be used as attractive plants for nucleation process during recover of disturbed sites in Atlantic Forest, favoring the local ecological succession.

\section{Acknowledgements}

To Léo Nascimento (Research manager from Itatiaia Nacional Park) to provide support and research permission, to the photographers Luiz Ribenboim (LR), Kacau Oliveira (KO), Francisco Falcon (FF) and Bertrando Campos (BC) for images and observations of animals. Credit of the images: LR - 3 (A), 4 (A, B, C, E, F, G), 5 (B, C, D, E, F, G, H), 6 (A, C, H), KO - 3 (G), 4 (D, H), 6 (B, D, E, F, G), BC - 3 (B), FF - 3 (I).

\section{References}

Albuquerque LB, Velazquez A \& Vasconcellos-Neto J (2006) Composição florística de Solanaceae e suas síndromes de polinização e dispersão de sementes em florestas mesófilas neotropicais. Interciência 31: 822-827.

Athiê S \& Dias MM (2012) Frugivoria por aves em um mosaico de Floresta Estacional Semidecidual e reflorestamento misto em Rio Claro, São Paulo, Brasil. Acta Botanica Brasilica 26: 84-93.

Aximoff I \& Freitas L (2009) Composição e comportamento de aves nectarívoras em Erythrina falcata (Leguminosae) durante duas florações consecutivas com intensidades diferentes. Revista Brasileira de Ornitologia 17: 194-203.

Aximoff I \& Freitas L (2010) Is pollen removal or seed set favored by flower longevity in a hummingbirdpollinated Salvia species? Annals of Botany 106: 413-419.

Bencke CS \& Morellato LPC (2002) Estudo comparativo da fenologia de nove espécies arbóreas em três tipos de floresta atlântica no sudeste do Brasil. Revista Brasileira de Botânica 25: 237-248. DOI: <http:// dx.doi.org/10.1590/S0100-84042002000200012>.

Bencke GA, Maurício GN, Develey PF \& Goerck JM (2006) Áreas importantes para a conservação das aves no Brasil, parte I - estados do domínio da Mata Atlântica. SAVE Brasil, São Paulo. 494p.

BFG - The Brazil Flora Group (2018) Brazilian Flora 2020: innovation and collaboration to meet Target 1 of the Global Strategy for Plant Conservation (GSPC). Rodriguésia 69: 1513-1527.

Cáceres NC \& Lessa LG (2012) O papel de marsupiais na dispersão de sementes. In: Cáceres NC (ed.) Os marsupiais do Brasil: biologia, ecologia e conservação. Ed. UFMS, Campo Grande. Pp. 407426.
Campos WH, Miranda-Neto A, Peixoto HJC, Godinho LB \& Silva E (2012) Contribuição da fauna silvestre em projetos de restauração ecológica no Brasil. Pesquisa Florestal Brasileira 32: 429-440.

Canela MBF (2006) Interações entre plantas e beijaflores em uma comunidade de Floresta Atlântica Montana em Itatiaia, RJ. Tese de Doutorado. Universidade Estadual de Campinas. Campinas. 75f.

Cavallero L, Raffaele E \& Aizen MA (2013) Birds as mediators of passive restoration during early post-fire recovery. Biological Conservation 158: 342-350.

CBRO - Comitê Brasileiro de Registros Ornitológicos (2014) Listas das aves do Brasil. 11 a edição. Disponível em $<$ http://www.cbro.org.br/>. Acesso em 25 janeiro 2014.

Ceccon E \& Varassin IG (2014) Plant-pollinator interactions in ecosystems restoration. In: Benítez M, Miramontes O \& Valiente-Banuet A (eds.) Frontiers in ecology, evolution and complexity. CopIt arXives, Mexico. 283p.

Cocucci A (2000) Evolutionary radiation in neotropical Solanaceae. Solanaceae IV: advances in biology and utilization. The Royal Botanic Gardens, Kew. Pp. 9-22.

Côrtes MC, Cazetta E, Staggemeier VG \& Galetti M (2009) Linking frugivore activity to early recruitment of a bird dispersed tree, Eugenia umbelliflora (Myrtaceae) in the Atlantic rainforest. Austral Ecology 34: 249-258.

Costa ADO, Salgado CM \& Dinali YT (2012) Caracterização da precipitação no médio vale do Rio Paraíba do sul Fluminense - RJ. Revista Geonorte 3: 1000-1013.

Cruz A (1981) Bird activity and seed dispersal of a montane forest tree (Dunalia arborescens) in Jamaica. Biotropica 13: 34-44.

Engriser EM (1995) The effect of insect larvae infestation on fruit choice in phyllostomid fruit bats: an experimental study. Biotropica 27: 523-525.

Fadini RF \& Marco JrP (2004) Interações entre aves frugívoras e plantas em um fragmento de mata atlântica de Minas Gerais. Ararajuba 12: 15-21.

Galetti M, Costa C \& Cazetta E (2003) Effects of forest fragmentation, anthropogenic edges and fruit color on the consumption of ornithocoric fruits. Biological Conservation 111: 269-293.

Galetti M, Laps R \& Pizo MA (2000) Frugivory by toucans (Ramphastidae) at two altitudes in the Atlantic Forest of Brazil. Biotropica 32: 842-850.

Hasui É, Souza V, Kiefer MC, Tamashiro J \& Silva WR (2009) Spatial and seasonal variation in niche partitioning between blue manakin (Chiroxiphia caudata) and greenish schiffornis (Schiffornis virescens) in southeastern Brazil. Studies on Neotropical Fauna and Environment 44: 149-159.

Hunziker AT (2001) The genera of Solanaceae. ARG Ganter Verlag K-G, Ruggell, Liechtenstein. 272p. 
ICMBio (2013) Plano de Manejo do Parque Nacional do Itatiaia. Encarte 3. ECOMEK, Brasília. 215p.

Jordano P, Galetti M, Pizo MA \& Silva WR (2006) Ligando frugivoria e dispersão de sementes à biologia da conservação. In: Rocha CFD, Bergallo HG, Sluys MV \& Alves MAS (eds.) Biologia da conservação: essências. RiMa, São Carlos. Pp. 411-436.

Levey DJ (1987) Seed size and fruit-handling techniques of avian frugivores. American Naturalist 129: 471485.

Malizia LR (2001) Seasonal fluctuations of birds, fruits, and flowers in a subtropical forest of Argentina. Condor 103: 45-61.

Manhães MA, Assis LCS \& Castro RM (2003) Frugivoria e dispersão de sementes de Miconia urophylla (Melastomataceae) por aves em um fragmento de Mata Atlântica secundária em Juiz de Fora, Minas Gerais, Brasil. Ararajuba 11: 173-180.

Moegenburg SM \& Levey DJ (2003) Do frugivores respond to fruit harvest? An experimental study of short-term responses. Ecology 84: 2600-2612.

Morellato LPC, Talora DC, Takahashi A, Bencke C C, Romero EC \& Ziparro VB (2000) Phenology of Atlantic rain forest trees: a comparative study. Biotropica 32: 811- 823 .

Morellato PC \& Leitão-Filho HF (1992) Padrões de frutificação e dispersão na Serra do Japi. In: Morellato PC (ed.) História Natural da Serra do Japi: ecologia e preservação de uma área florestal no sudeste do Brasil. UNICAMP/FAPESP, Campinas. Pp. 112-140.

Newstrom LE, Frankie GW \& Baker HG (1994) A new classification for plant phenology based in flowering patterns in lowland tropical rain forest trees at La Selva, Costa Rica. Biotropica 26: 141159. DOI: <http://dx.doi.org/10.2307/2388804>.

Novoa S, Cadenillas R \& Pacheco V (2011) Dispersión de semillas por murciélagos frugívoros en bosques del Parque Nacional Cerros de Amotape, Tumbes, Perú. Mastozoología neotropical 18: 81-93.

Oliveira-Filho AT \& Fontes MAL (2000) Patterns of floristic differentiation among Atlantic Forests in Southeastern Brazil and the Influence of Climate1. Biotropica 32: 793-810.

Parrini R \& Pacheco JF (2011a) Frugivoria por aves em Alchornea triplinervia (Euphorbiaceae) na Mata Atlântica do Parque Estadual dos Três Picos, estado do Rio de Janeiro, Brasil. Atualidades Ornitológicas On-Line 162: 33-41.

Parrini R \& Pacheco JF (2011b) Frugivoria por aves em seis espécies arbóreas do gênero Miconia (Melastomataceae) na Mata Atlântica do Parque Nacional da Serra dos Órgãos, Região Sudeste do Brasil. Atualidades Ornitológicas 159: 51-58.

Parrini R \& Pacheco JF (2014) Aspectos da frugivoria por aves em Cupania oblongifolia (Sapindaceae) na Mata Atlântica do Parque Nacional da Serra dos Órgãos, estado do Rio de Janeiro, Brasil. Atualidades Ornitológicas 178: 55-62

Parrini R, Pacheco JF \& Haefeli L (2008) Observação de aves se alimentando dos frutos de Miconia sellowiana (Melastomataceae) na Floresta Atlântica Alto-Montana do Parque Nacional da Serra dos Órgãos e do Parque Nacional do Itatiaia, Região Sudeste do Brasil. Atualidades Ornitológicas 146: 4-7.

Peel MC, Finlayson BL \& Mcmahon TA (2007) Updated world map of the Köppen-Geiger climate classification. Hydrology and Earth System Sciences 11: 1633-1644.

Pereira TS \& Mantovani W (2007) Fenologia reprodutiva de Miconia cinnamomifolia (DC.) Naudin (Melastomataceae), em floresta submontana no estado do Rio de Janeiro. Revista de Biologia Neotropical 4: 31-45.

Pineschi RB (1990) Aves como dispersores de sete espécies de Rapanea (Myrsinaceae) no maciço do Itatiaia, estados do Rio de Janeiro e Minas Gerais Ararajuba 1: 73-78.

Pizo MA (2012) O movimento dos animais frugívoros e das sementes em paisagens fragmentadas. In: Del-Claro K \& Torezan-Silingardi HM (orgs.) Ecologia das interações plantas-animais: uma abordagem ecológicoevolutiva. Technical Books, Rio de Janeiro. Pp. 143-157.

Pizo MA (2007) Frugivory by birds in degraded areas of Brazil. In: Dennis AJ, Schupp EW, Green RJ \& Westcott DW (eds.) Seed dispersal: theory and its application in a changing world. CABI Publishing, Wallingford. Pp. 91-110.

Pizo MA \& Galetti M (2010) Métodos e perspectivas do estudo da frugivoria e dispersão de sementes por aves. In: Matter SV, Straube FC, Accordi IA, Piacentini VQ \& Cândido-Jr JF (orgs.) Ornitologia e conservação: ciência aplicada, técnicas de pesquisa e levantamento. Technical Books, Rio de Janeiro. Pp. 493-503.

Reis A, Zambonin RM \& Nakazono EM (1999) Recuperação de áreas florestais degradadas utilizando a sucessão e as interações plantaanimal. Série Recuperação, Caderno 14. Conselho Nacional da Reserva da Biosfera da Mata Atlântica, São Paulo. 42p.

Ridgely RS, Gwynne JA, Tudor G \& Argel M (2015) Aves do Brasil: Mata Atlântica do Sudeste. Wildlife conservation Society. Editora Horizonte, São Paulo. 432p.

Segadas-Vianna F \& Dau L (1965) Ecology of Itatiaia range, southeastern Brazil. II. Climates and altitudinal climatic zonation. Arquivos Museu Nacional Rio de Janeiro 53: 31-53.

SEMA - Secretaria do Meio Ambiente do Estado do Rio de Janeiro (1998) Lista das espécies ameaçadas 
de extinção do estado do Rio de Janeiro. PortariaSEMA n ${ }^{\circ} 001$, de 4 de junho de 1998 - RJ.

Sick H (1997) Ornitologia Brasileira. Edição revista e ampliada por Pacheco JF. Editora Nova Fronteira, Rio de Janeiro. 862p.

Silva JMC, Sousa Mc \& Castelletti CHM (2004) Areas of endemism for passerine birds in the Atlantic Forest. Global Ecology and Biogeography 13: 85-92.

Silva WR, de Marco P, Hasui E \& Gomes VSM (2002) Patterns of fruit-frugivore interactions in two Atlantic forest bird communities of southeastern Brazil: implications for conservation. In: Levey DJ, Silva WR \& Galetti M (eds.) Seed dispersal and frugivory: ecology, evolution and conservation. CABI International, Wallingford. Pp. 423-435.

Smith SD, Ané C \& Baum DA (2008) The role of pollinator shifts in the floral diversification of Iochroma (Solanaceae). Evolution 62: 793-806.

Souza TO \& Vilela DAR (2013) Espécies ameaçadas de extinção vítimas do tráfico e criação ilegal de animais silvestres. Atualidades Ornitológicas 176: 64-68.

Van der Pijl L (1982) Principles of seed dispersal in higher plants. Spring-Verlag, New York. 218.
Verçoza FC, Dias AR \& Missagia CCC (2012) Ecologia da polinização e potenciais dispersores da "marianeira" Acnistus arborescens (L.) Schltdl. (Solanaceae) em área de Floresta Atlântica do Rio de Janeiro. Natureza on line 10: 59-64.

Vogel HF, Campos JB \& Bechara FC (2015) Early bird assemblages under different subtropical forest restoration strategies in Brazil: passive, nucleation and high diversity plantation. Tropical Conservation Science 8: 912-939.

Wheelwright NT, Haber WA, Murray KG \& Guindon C (1984) Tropical fruit-eating birds and their food plants: a survey of a Costa Rican lower montane forest. Biotropica 173-192.

Wolowsky M \& Freitas L (2010). Sistema reprodutivo e polinização de Senna multijuga (Fabaceae) em Mata Atlântica Montana. Rodriguésia 61: 167-179.

Wolowski M, Ashman T-L \& Fretas L (2013a) Community-wide assessment of pollen limitation in hummingbird-pollinated plants of a tropical montane rain forest. Annals of Botany 112: $903-$ 910.

Wolowski M, Saad CF, Ashman T-L \& Fretas L (2013b) Predominance of self-compatibility in hummingbird-pollinated plants in the Neotropics. Naturwissenschaften 100: 69-79. 